\title{
Echo width of foam supports used in scattering measurements
}

\section{Appel-Hansen, Jørgen; Solodukhov, V. V.}

\section{Published in:}

I E E E Transactions on Antennas and Propagation

Publication date:

1979

Document Version

Publisher's PDF, also known as Version of record

Link back to DTU Orbit

Citation (APA):

Appel-Hansen, J., \& Solodukhov, V. V. (1979). Echo width of foam supports used in scattering measurements. I E E E Transactions on Antennas and Propagation, 27(2), 191-193.

\section{General rights}

Copyright and moral rights for the publications made accessible in the public portal are retained by the authors and/or other copyright owners and it is a condition of accessing publications that users recognise and abide by the legal requirements associated with these rights.

- Users may download and print one copy of any publication from the public portal for the purpose of private study or research.

- You may not further distribute the material or use it for any profit-making activity or commercial gain

- You may freely distribute the URL identifying the publication in the public portal

If you believe that this document breaches copyright please contact us providing details, and we will remove access to the work immediately and investigate your claim 


\title{
Echo Width of Foam Supports Used in Scattering Measurements
}

\author{
J $\emptyset$ RGEN APPEL-HANSEN, MEMBER, IEEE, AND V. V. SOLODUKHOV
}

\begin{abstract}
Theoretically and experimentally determined echo widths of dielectric cylinders having circular, triangular, and quadratic cross sections have been compared. The cylinders were made of foam material having a relative dielectric constant of about 1.035 . The purpose of the investigation was to find a cross section with a small echo width. The result can be used in selecting a proper support for test objects in backscattering measurement studies.
\end{abstract}

\section{INTRODUCTION}

$I^{1}$ N SCATTERING and antenna measurements it is desirable to have supports with minimum influence on the test object [1], [2]; i.e., scattering from the support is at a minimum. The most widely used supports are foamed plastic pedestals. In order to reduce scattering the supports are often shaped as a truncated cone having a circular cross section [3]. Due to its rotational symmetry this type of cross section provides a support which can be used in many types of measurement programs. The purpose of this work is to investigate whether other types of cross sections may be better suited for specific scattering studies. The present paper will concentrate on investigating supports for backscattering studies where it is wanted to measure scattering at one aspect angle of the test object at a time. For such measurements a promising cross section seems to be one having a wedge that can be positioned to point in the dircction of the scattering measurement setup. To' minimize interaction between the support and test object, this may be positioned on top of the support close to the tip of the wedge. Since a practical wedge cannot be extended infinitely in the direction away from the tip, the wedge has to be terminated with a back wall. In case a flat wall is chosen perpendicular to the setup, an isosceles triangular cross section is created. However, as will be demonstrated, this type of back wall gives rise to a larger reflection than can be expected from a back wall which is also formed like a wedge. From the above considerations it was decided to compare right cylinders having circular, triangular, and square cross sections. Since a wedge with an angle of $90^{\circ}$ is most easily manufactured, the simple cross sections having the same maximum diameter $d$ as shown in Fig. 1(a) were investigated. The reader may imagine other cross sections and other directions of incidence which would be worthwhile to investigate. In order to facilitate such studies and describe the computations given in this paper, the theory for determining the echo width of cylinders of arbitrary cross sections as shown in Fig. 1(b) is outlined in Section II. In Section III the experimental procedure is described. Theoretical and experimental results are presented in Section IV where

Manuscript received December 1, 1977; revised June 12, 1978.

J. Appel-Hansen is with the Radio Anechoic Chamber, Electromagnetics Institute, Technical University of Denmark, Lyngby, Denmark.

V. V. Solodukhov is with the Moscow Power Institute, Moscow, U.S.S.R. During the measurement work, he visited the Radio Anechoic Chamber in connection with a stay at the Institute of Electronic Systems, Aalborg University Center, Aalborg, Denmark.

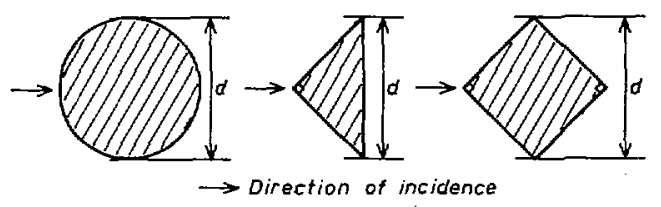

(a)

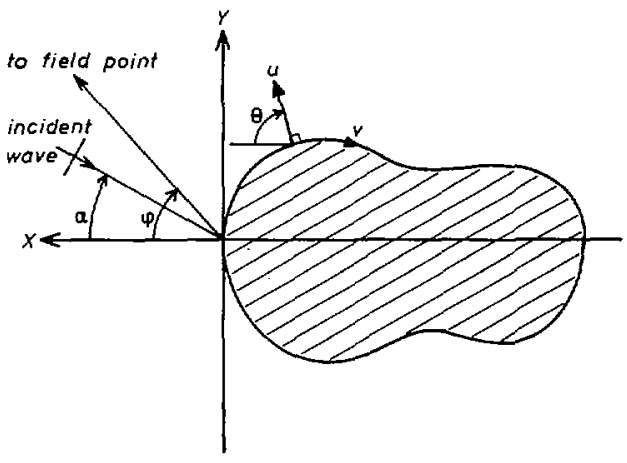

(b)

Fig. 1. Cross section of cylinder. (a) Investigated cross sections. (b) Coordinate systems relative to an arbitrary cross section.

conclusions are drawn concerning the best suited support for specific purposes.

In actual measurements supports are not of infinite length but usually extend from the middle of the test range down to the ground level where they may be mounted on an azimuth positioner. However, although our theoretical model is rather different from actual supports, the conclusions of this work are expected to be pertinent to scattering work and have in fact proved to be valuable in our latest measurement programs.

\section{THEORY}

Electromagnetic scattering by a homogeneous dielectric cylinder of arbitrary cross section can be investigated by solving integral equations. Two approaches exist. The equations can be written in terms of polarization currents [4], [5] or surface currents [6]-[8]. In the first approach twodimensional integral equations over the cross section of the cylinder have to be solved. This approach requires the inversion of a large full matrix which is numerically impractical for cylinders of a large cross-sectional area. In the present investigation, where the maximum dimension of the cylinder is a few wavelengths, the second approach, which uses one-dimensional integral equations containing integrals over the contour of the cross section, is applied.

Let the axis of an infinitely long cylinder be placed parallel to the $z$-axis of a rectangular $x y z$-coordinate system. The cylinder may have an arbitrary cross section in the $x y$-plane as shown in Fig. 1(b). The only restriction is that an orthogonal cylindrical $u v z$-coordinate system with a surface $u=u_{0}=$ 
constant coinciding with the surface of the cylinder can be introduced. The cylinder is dielectric having a relative permeability equal to one and a relative dielectric constant which is denoted $\epsilon$.

By using standard procedures the system of integral equations given in [6] is reduced to a system of linear equations in terms of the electric and magnetic surface currents at discrete sampling points of the contour. In the case of $E$-polarization the scattered field $E_{z}{ }^{\text {sc }}$ at large distances $(r \gg 1)$ can be written as

$$
E_{z} \mathrm{sc}(r, \phi)=\sqrt{\frac{2 i}{\pi r}} e^{-i r} g(\phi)
$$

where

$$
g(\phi)=\frac{1}{4} \int_{v}\left[\eta K_{v} \cos (\theta-\phi)-J_{z}\right] e^{i(x \cos \phi+y \sin \phi)} d v
$$

and the direction to the field point is characterized by the angle $\phi$ as shown in Fig. 1(b). $(x, y)$ are the coordinates of the point of integration $\left(u_{0}, v\right)$ and $\theta$ is the angle between the $x$ axis and the outgoing normal to the cylinder at $\left(u_{0}, v\right) . \eta$ is the free-space admittance, $K_{v}$ is the $v$-component of the magnetic current, and $J_{z}$ is the $z$-component of the electric current. To be consistent with [6], the time factor is $e^{i \omega t}$, and all linear quantities are multiplied by the free-space wavenumber $k_{0}$. Further details may be found in [9].

The echo width $w_{e}(\phi)$ of the cylinder is defined as

$$
w_{e}(\phi)=\lim _{r \rightarrow \infty} 2 \pi r\left|\frac{E_{z} \mathrm{sc}(r, \phi)}{E_{z}^{\text {inc }}}\right|^{2},
$$

where $E_{z}$ inc is the electric-field intensity of the incident wave. Introducing $k_{0}$ in (1) and (2) we obtain

$$
w_{e}(\phi)=\frac{4}{k_{0}}|g(\phi)|^{2}
$$

In the following sections it is often convenient to consider the behavior of $w_{e} k_{0}$.

Although the developed computer program is able to calculate the scattered field from cylinders of arbitrary shapes for both $E$ - and $H$-polarizations, the present investigation is limited to compare backscattered fields $(\alpha=\phi)$ for $E$-polarization in the practical cases of cylinders having simple circular, triangular, and square cross sections having the same maximum dimension $d$ as shown in Fig. 1(a). Furthermore, in the case of the triangular and square cylinders the incident wave is propagating along a line symmetry as indicated in Fig. 1(a); i.e., the "wedge-on" echo width of a rectangular wedge terminated in two manners is computed. In Section IV measured results confirm the validity of the theoretical approach.

\section{EXPERIMENTAL SETUP AND PROCEDURES}

The experiments were carried out in a radio anechoic chamber. In previous measurement programs it was demonstrated that results obtained in such a three-dimensional test range can be compared with theoretical results calculated for the two-

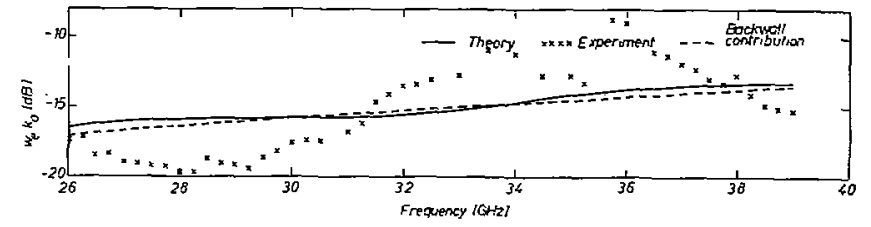

Fig. 2. Echo width of triangular cylinder.

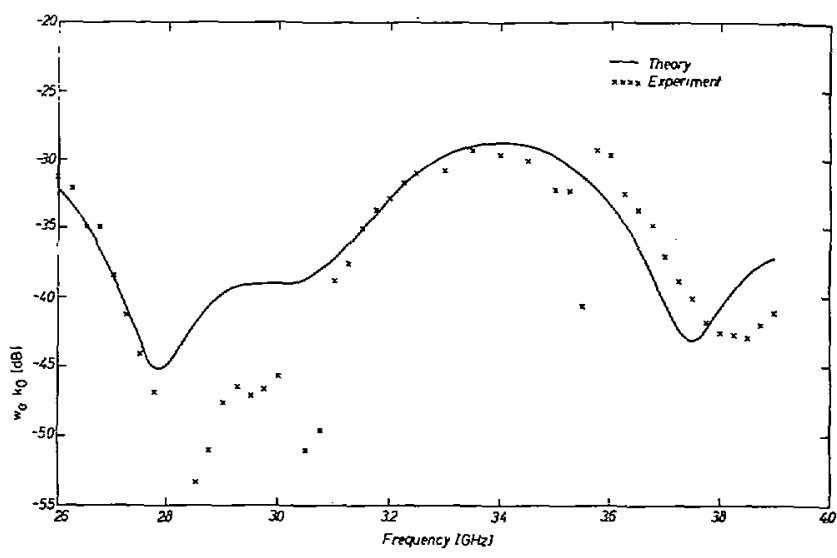

Fig. 3. Echo width of square cylinder.

dimensional case [10], [11]. The condition for accurate measurements is that the cylinder is sufficiently long so that relatively narrow-beam antennas do not significantly illuminate the ends of the cylinder. Then the scattering properties of a finite cylinder may be simply related to those of an infinitely long object. In the present experiments the cylinders were 3-m long and the distance to the radar setup was $9.7 \mathrm{~m}$. The largest dimension of the cross section of the cylinders was $30 \mathrm{~cm}$. The radar was a nearly monostatic radar setup. Further information on the experimental setup and procedures may be found in [1] and [10].

\section{RESULTS}

In Figs. 2 and 3 the echo widths (multiplied by the freespace propagation constant) of the triangular and square cylinders, respectively, are shown. The theoretical curves were obtained from the theory given above. The experimental results were obtained by using the circular cylinder as a reference object. Before comparing the results it is convenient to note that $w_{e}$ of the circular cylinder versus frequency varies approximately like a standing-wave curve with a standing-wave ratio equal to infinity. This is because the backscattering of a circular cylinder is due mainly to the interference of reflections of approximately the same magnitude from the front and rear surfaces. Thus as frequency varies, the two reflections add in and out of phase. The frequency spacing $\Delta f$ between successive minima is determined approximately by

$$
\Delta f=\frac{c}{2 d \sqrt{\epsilon}},
$$

where $c$ is the velocity of light in free space and $d$ is the diameter [3]. In the actual measurements it was observed that $w_{e}$ of the circular cylinder tended to zero at two frequencies within the investigated interval from 2.6 to 3.9 $\mathrm{GHz}$. By inserting the spacing between the two frequencies into (5) the dielectric constant $\epsilon$ of the material was found approximately to be 1.035 . 
As it appears from Fig. $2, w_{e} k_{0}$ as a function of $f$ for the triangular cylinder is nearly constant. In fact, computations show that $w_{e} k_{0}$ increases about $2 \mathrm{~dB}$ as the frequency is increased. The degree of constancy of $w_{e} k_{0}$ is because the back wall gives the major contribution which is proportional to the square of the coefficient of reflection $R$, and the edges give a minor contribution [12]-[14]. The contribution from the back wall can be calculated by using a physical-optics approximation. Considering the back wall to be an infinitely long strip with a surface current distribution, (2) and (3) give small values for $\phi$ and $\alpha=0$

$$
w_{e}(\phi) \cong \frac{\left(2 k_{0} a\right)^{2}}{k_{0}} R^{2} \frac{\sin ^{2}\left(k_{0} a \sin \phi\right)}{\left(k_{0} a \sin \phi\right)^{2}},
$$

where $2 a=d$ is the width of the back wall. This equation is equivalent to analogous results found in standard textbooks [15]. As shown in Fig. 2, the back-wall contribution based on (6) shows agreement within $0.5 \mathrm{~dB}$ for the actual cylinder.

For the square cylinder, the behavior of $w_{e} k_{0}$ is shown in Fig. 3. For the direction considered geometrical optics show that out of the four walls only the two back walls contribute. In fact, these make the configuration of a corner reflector. Thus the contribution from the walls is proportional to $R^{4}$. The fields diffracted by the edges are of the same order. Therefore, the interference between these two components results in the irregular variations in Fig. 3. It should be mentioned that by choosing a quadrangle having angles different from $90^{\circ}$, it is expected that the echo width may be further reduced owing to the lack of the corner reflector effect just mentioned.

Noting that the maximum value of $w_{e} k_{0}$ of the circular cylinder is calculated to be $-19.6 \mathrm{~dB}$, it is seen that the maximum value of $w_{e} k_{0}$ for the square cylinder is $9.2 \mathrm{~dB}$ lower than that obtained for the circular cylinder. Furthermore, the maximum value of the triangular cylinder is $6.3 \mathrm{~dB}$ larger than that of the circular cylinder. Thus in general the square cylinder is the best suited for backscattering measurements. Only in narrow frequency bands around the minima in the standing wave pattern of $w_{e} k_{0}$ for the circular cylinder may this be the best support.

These conclusions are based on the agreement between computed and measured values which is often better than $1 \mathrm{~dB}$. One of the major sources of error is that the measurements were carried out with a nearly monostatic radar having an inherent bistatic angle of about $2.2^{\circ}$. Another source of error was that the value of $\epsilon$ was not known beforehand and was determined as described above. The major discrepencies are observed around the frequencies 3.1 and $3.6 \mathrm{GHz}$. This is due to the use of the circular cylinder as a reference object having low values of $w_{e} k_{0}$ around these frequencies. Further details may be found in [9].

\section{CONCLUSIONS}

The backscattering echo widths of circular, triangular, and square cylinders have been compared. It is concluded that in general the square is the best suited for backscattering measurements at fixed aspect angles. This is due to the observation that the square cross section has an echo width which in average is more than $10 \mathrm{~dB}$ below that of the circular cross section. Only for narrow frequency band uses can the diameter of the circular cross section be chosen to have a lower echo width.
During the investigation it was observed that the triangular cylinder, due to its back wall, has a relatively high echo width which increases slowly with frequency. Furthermore, the echo width of the triangular cylinder may be calculated accurately by taking only the back wall into account. Therefore, the triangular cylinder might easily be used as a reference. Comparison between the echo width of circular and triangular cylinders might be used to find the dielectric constant of the material. Further investigation of the accuracy of such a procedure would be worthwhile to initiate.

\section{ACKNOWLEDGMENT}

The authors wish to thank Lars Hougaard who performed most of the measurement work. Thanks are also due to Jørgen Bach Andersen for commenting on the manuscript.

\section{REFERENCES}

[1] J. Appel-Hansen, "Precision measurement of backscattering cross-sections as a function of frequency," IEEE Trans. Instrum. Meas., vol. IM-25, pp. 363-370, Dec. 1976.

[2] J. Appel-Hansen and N. Burke, "Supports for antenna radiation pattern measurements," Electromagnetics Inst., Tech. Univ. of Denmark, Lyngby, R 185, July 1977.

[3] T. B. A. Senior, M. A. Plonus, and E. F. Knott, "Designing foamed-plastic target supports," Microwaves, pp. 38-43, Dec. 1964.

[4] J. H. Richmond, "Scattering by a dielectric cylinder of arbitrary cross-section shape," IEEE Trans. Antennas Propagat., vol. AP-13, pp. 334-341, May 1965.

[5] -, "TE-wave scattering by a dielectric cylinder of arbitrary cross-section shape," IEEE Trans. Antennas Propagat., vol. AP-14, pp. 460-464, July 1966.

[6] V. V. Solodukhov and E. N. Vasil'ev, "Diffraction of a plane electromagnetic wave by a dielectric cylinder of arbitrary crosssection," Soviet Phys. Tech. Phys., vol. 15, no. 1, pp. 32-36, July 1970; also in Zhurnal Tekhnicheskoi Fiziki, yol. 40, no. 1, pp. 47-53, Jan. 1970.

[7] E. N. Vasil'ev and V. V. Solodukhov, "Oblique incidence of electromagnetic wave on a dielectric cylinder with arbitrary crosssection shape," in Computing Methods and Programing (in Russian). Moscow, U.S.S.R.: MGU, 1973, no. 20, pp. 144-157.

[8] N. Morita, "Surface integral representations for electromagnetic scattering from dielectric cylinders," IEEE Trans. Antennas Propagat., vol. AP-26, pp. 261-266, Mar. 1978.

[9] J. Appel-Hansen and V. V. Soldoukhov, "Comparison of echo width of foam supports used in scattering measurements," Electromagnetics Instit. Tech. Univ. of Denmark, Lyngby, R 197, May 1978.

[10] H. E. Green and J. Appel-Hansen, "Echo width of axially slitted hollow cylinders," Electromagnetics Inst., Tech. Univ. of Denmark, Lyngby, R 153, Jan. 1976.

[11] J. Appel-Hansen and W. V. T. Rusch, "Dynamic measurements of forward scattering," IEEE Trans. Antennas Propagat., vol. AP-23, pp. 846-850, Nov. 1975.

[12] E. N. Vasil'ev and V. V. Solodukhov, Annotations of Papers Read at the Fifth All-Union Symposium on Diffraction and Propagation Waves (in Russian). Leningrad, U.S.S.R.: LGU, 1970 , p. 10.

[13] -, "Numerical solution of the problem of electromagnetic diffraction by a dielectric wedge," in Proc. Int. Symp. on the Theory of Electromagnetic Waves, Tbilisi, Sept. 1971; also in Electromagnetic Wave Theory (Preprints). Moscow, U.S.S.R.: Nauka, 1971, pp. 512-516.

[14] - "Diffraction of electromagnetic waves by a dielectric wedge," Radiophys. Quantum Electron., pp. 1161-1169, Mar. 10, 1976; also in Izvestiya VUZ, Radiofizika, vol. 17, no. 10, pp. 1518-1528, Oct. 1974.

[15] J. W. Crispin, Jr., and K. M. Siegel, Methods of Radar Cross-Section Analysis. New York: Academic Press, 1968, p. 122. 